\title{
Research on key technology of Mining Remote Sensing Dynamic Monitoring Information System
}

\author{
Jiuhu Sun, Hengmao Xiang \\ Shandong Provincial Institute of Land Surveying and Mapping, Jinan,250102, China-(sunjhgis,hmxiang2003)@ 126.com
}

KEY WORDS: Mining, Remote Sensing, dynamic monitoring, mobile GIS , tiantidu

\begin{abstract}
:
Problems exist in remote sensing dynamic monitoring of mining are expounded, general idea of building remote sensing dynamic monitoring information system is presented, and timely release of service-oriented remote sensing monitoring results is established. Mobile device-based data verification subsystem is developed using mobile GIS, remote sensing dynamic monitoring information system of mining is constructed, and "timely release, fast handling and timely feedback" rapid response mechanism of remote sensing dynamic monitoring is implemented.
\end{abstract}

\section{INTRODUCTION}

Mineral resources are an important material basis of China's social and economic development. For a long time, development and utilization of mineral resources have been relatively extensive in China due to various reasons. There are still some prominent problems exist in management of mineral resources development, and mine exploitation has caused a serious waste. The Party Central Committee and the State Council attach importance to the protection and rational development and utilization of mineral resources, and have made comprehensive efforts to rectify and standardize the order of mineral resources development. Compared with conventional means, remote sensing technology performs detection in a high-altitude aerial view way, which can surmount traffic barriers and visual limits; remote sensing dynamic monitoring can quickly and duly provide relevant departments with timely and objective basic data and decision-making basis for exercise of governmental functions and rectification of mining development order, can enhance the high-tech content in supervision and management, and can improve the efficiency of supervision and management and the quality of management; meanwhile, mineral resources exploitation remote sensing monitoring has also formed a relatively mature technological roadmap (Datcu,2003; Nie Hongfeng,2007; Chen Weitao,2009; Nan Junxiang,2012; Wang Yonggang,2008). Shandong Province has been carrying out mining remote sensing monitoring pilot work since 2006 to provide scientific and reliable information for mineral resources regulation utilizing the advanced, broad and time-sensitive characteristics of remote sensing technology. At present, remote sensing dynamic monitoring mechanism has been formed for open pit mines in Shandong Province's bedrock areas, which provides land and resources departments with scientific law enforcement basis for the development and management of mineral resources, as well as low-cost, fast and efficient fight against illegal mining behavior.

1.1 Introduction of Mineral Resources Remote Sensing Dynamic Monitoring Project in Shandong Province

In 2006, in Linyi City, Shandong Province launched a pilot remote sensing dynamic monitoring project in Linyi City's Lanling County (formerly Cangshan County)( Cao
Hongsong,2007). After achieving success, Shandong Province has carried out multi-round monitoring in 93 counties (cities and districts) of the province successively. Through monitoring and field verification statistics, the distribution status and property of unlicensed mining and patch mining in the monitoring zone have been identified, and cross-border mining, mining instead of exploration and other illegal phenomena are found timely, thereby providing comprehensive and accurate first-hand information and basis for mining administration and management departments' management and law enforcement.

\subsection{Existing Problems}

Shandong Province has carried out five rounds of remote sensing dynamic monitoring in open pit mines in the entire province's bedrock areas, and has accumulated a wealth of experience. However, the intermittent uncertainty of illegal mining, the variability of patch mining, and the "guerrilla" character and repeatability of covert mining have led to the following problems in the remote sensing dynamic monitoring work:

i) Untimely release of remote sensing monitoring results.

Due to the large scope of monitoring area, remote sensing image processing and patch interpretation workloads are large. As remote sensing monitoring results are distributed to cities and counties in the traditional way of map printing, suspected illegal patches cannot be found and verified timely. ii) The poor quality of data verification.

In accordance with the existing mining administration and management system, suspected illegal patch mining shall be verified and handled by local mining administration and management authorities. In the actual practice, superior authorities can not verify the authenticity and accuracy of data reported by cities and counties due to the low verification quality of primary-level staff and other problems, leading to ineffective supervision of local land and resources departments, thus resulting in mine mismanagement.

Based on the above background, this paper presents ideas such as implementation of rapid monitoring data release by service mode and improvement of field verification data quality through the practice of remote sensing dynamic monitoring project in Shandong Province's bedrock areas, and completes the development of mining remote sensing dynamic monitoring information system. 


\section{DESIGN IDEAS}

\subsection{Quick Release of Monitoring Data}

The remote sensing dynamic monitoring project of open pit mines in Shandong Province's bedrock areas has a large monitoring range, which involves 13 cities. Monitoring data include monitoring images and interpretation patches. Monitoring images are sliced in accordance with Platform for geo-information common services Data specification for electronic map(National Geomatics Center of China,2011), and published as OGC WMTS services; while interpretation patches are published as OGC WMS/WFS services. The above service through the MAPWORLD service registration, monitoring data is achieved based on network for everyone, as shown in Figure 1.

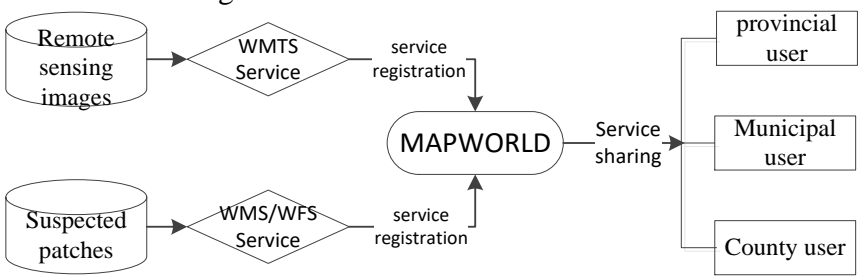

Figure 1. Flow chart of monitoring data releasion

\subsection{Mobile Terminal-based Data Verification Technology}

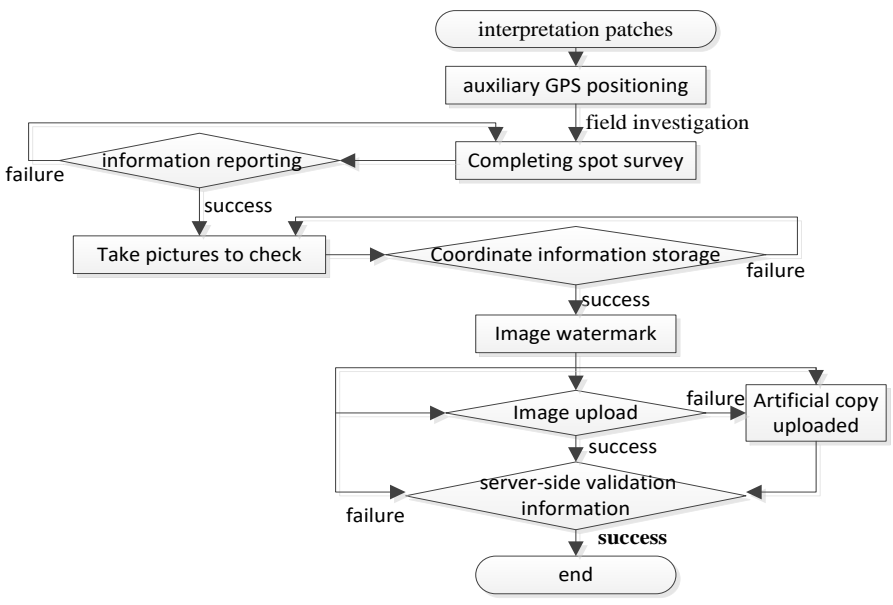

Figure 2. Flow chart of data verification technology

Mobile GIS(Yu Tao,2002) has mobile, dynamic and locationdependent features(Zhang Ergang,2013). This paper achieves the field data verification of mine interpretation patches based on the mobile GIS technology using terminals such as smart phone and tablet PC. Currently, Apple's iPad device is characterized by strong screen information carrying capacity, high system controllability and excellent battery life $(\mathrm{Xu}$ Liuhua,2012), so field verification of monitoring patches is implemented using iPad in this paper, and data verification quality is improved in two aspects: Firstly, verification routes are obtained based on GPS information for verification of whether the inspectors have arrived at the patch location. Secondly, GPS information is stored in the Exchangeable Image File (Exif) format(Li Yao,2012) when shooting field patch pictures, moreover, the pictures are watermarked with coordinates and time information for verification of authenticity of pictures.
Flow of mobile terminal-based data verification technology is shown in Figure 2.

\section{OVERALL SYSTEM FRAMEWORK}

Based on the above ideas, remote sensing dynamic monitoring and management information system of mining is built using service-oriented architecture (SOA), as shown in Figure 3 .

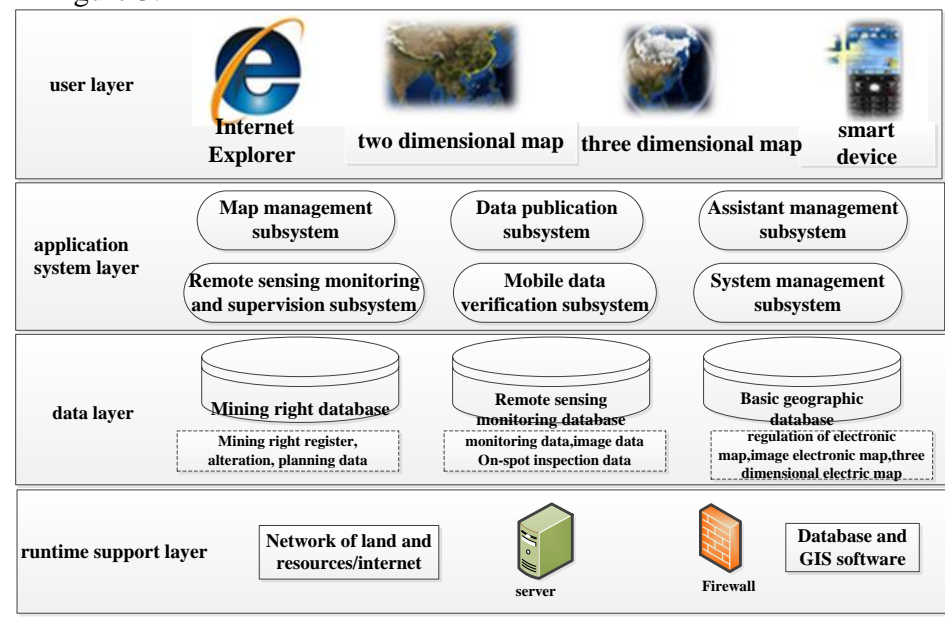

Figure 3. Overall architecture diagram of system

The system is divided into runtime support layer, data layer, application system layer and user layer. Networks as well as software and hardware environments supporting system running are constructed relying on the land and resources intranet and the Internet. Mineral rights database and remote sensing dynamic monitoring database are built using Oracle and ArcSDE software. Subsystems of map management, data publication, assistant management, remote sensing monitoring and supervision, mobile data verification and system management are created using the online geographic information services provided on the Geographic Information Public Service Platform of Shandong Province. Users use the system via $\mathrm{B} / \mathrm{S}$ access or mobile terminals, and smart devicebased verification and reporting mechanism is established. All of these help establish and improve the normalized mineral exploitation supervision system of Shandong Province with timely discovery, clear responsibility, powerful handling and effective supervision features.

\section{TECHNOLOGY IMPLEMENTATION}

Genetic algorithm is widely used in the multi-objective optimization problems with global search capability and robustness[7]. In order to solve the problem (3), we encode the upper variable values using real coding scheme, and give a fitness function based on non-dominated solutions sorting method and crowding distance, it can distinguish different individuals effectively. Our algorithm is developed:

\subsection{Database}

i) Database of mineral rights

By the end of 2014, there are a total of 3,014 mining rights and 872 prospecting rights in Shandong Province. Mineral rights database includes the following.

Mining rights data: integration of the province's mining registration data, including the ministry, province, city and county-issued mining rights. 
Prospecting rights data: integration of the province's prospecting registration data, including the ministry and province-issued prospecting rights.

Planning and geological data: integration of the province's mineral resources planning and geological data for assisting the competent authorities in setting up or managing the mineral rights.

ii) Remote sensing monitoring database

Integration of the province's remote sensing monitoring data over the years, which includes the following:

Remote sensing images: integration of remote sensing images in each round of dynamic monitoring.

Suspected patches: integration of patches in each round of monitoring, as well as field verification information of patches (including verification registration cards and field verification pictures). Field verification pictures are stored in the Exif JPEG format, and organized according to the administrative division code with patch as the unit in a "province codelverificationlcounty codelpatch numberlpicture" way.

Handling and execution status: legal handling and execution information on verified patches stored in the database, including legal handling and settlement results, statistical forms, etc.

iii) Geographic information

Online geographic information services provided on the Geographic Information Public Service Platform of Shandong Province are used, including the province's vector electronic map, image electronic map and three-dimensional electronic map. And geographic information services represented primarily by two-dimensional and threedimensional maps are implemented.

Based on the province's basic geographic information, the Geographic Information Public Service Platform of Shandong Province provides functional service interfaces, such as geographic name and address query, address coding and route analysis services. The system integrates the above functional services, thereby enhancing its usability, and assisting users in field verification.

\subsection{Software System}

The remote sensing dynamic monitoring and management information system of mining in Shandong Province establishes the entire operation flow of data publication, verification, handling and supervision based on remote sensing dynamic monitoring on the ground of open pit mines in the province's key areas to improve the "fast data acquisition, timely target detection, fast handling and timely feedback" rapid response mechanism of dynamic remote sensing monitoring. The system consists of five subsystems: map management subsystem, data publication subsystem, assistant management subsystem, remote sensing monitoring and supervision subsystem, and mobile data verification subsystem.

i) Map management subsystem

This subsystem provides common map and GIS functions, including map operation, space measurement, layer management, 3D viewing, contrast viewing, coordinate positioning and map labeling functions.

ii) Data publication subsystem

This subsystem changes the traditional paper-based data distribution mode. Monitoring organizations will use ArcGIS Server to release suspected patches and remote sensing images, in order achieve rapid release of remote sensing data for the use of various cities and counties within the province.

iii) Assistant management subsystem
This subsystem implements functions such as property query, spatial query and statistical analysis of prospecting and mining rights based on the province's mineral rights database. iv) Mobile data verification subsystem

Mobile data verification subsystem is developed based on the mobile GIS technology. Remote sensing monitoring data of relevant administrative zone can be used online or downloaded offline using iPAD device. Based on the GPS information, fast positioning of patch location is implemented, as well as field verification of patch information, filling of Report on Verification of Mineral Resource Satellite Image Patches and taking of field pictures (automatically records the coordinates of photographing sites, and adds watermarks), in order to facilitate users to quickly verify and handle illegal patches, thereby improving the accuracy and authenticity of information verification, as shown in Figure.4.

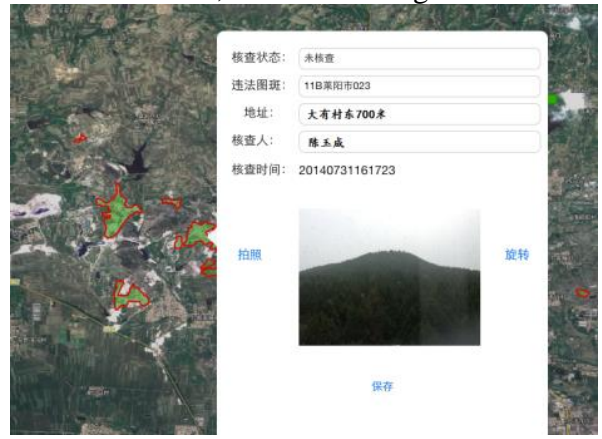

Figure 4. Functional interface of mobile data verification subsystem

v) Remote sensing monitoring and supervision subsystem This subsystem implements functions such as patch management, patch verification information management and implementation feedbacks and summary statistics based on provinces' remote sensing dynamic monitoring database.

As shown in Figure.5, the system displays the patch verification information on the image map for checking and reporting of information.

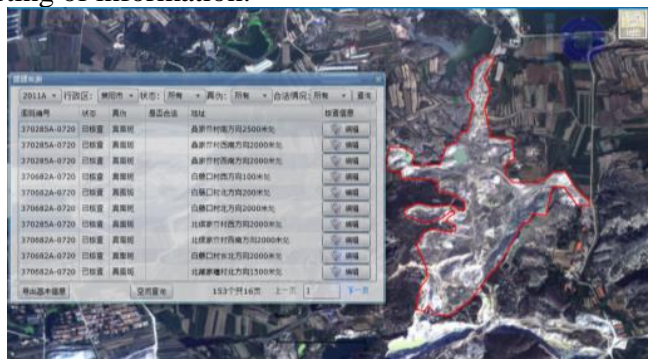

Figure 5. Management interface of patch verification information

Based on the province's patch verification information, the system can generate the illegal mining distribution hotspot map of the province as shown in Figure.6. The system can also generate the illegal mining prone spot map of the province utilizing the monitoring data over the years, as shown in Figure.7. 


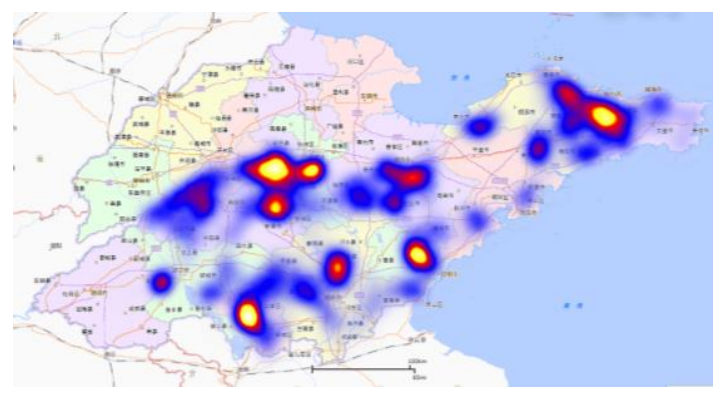

Figure 6. Map of illegal mining distribution hotspots

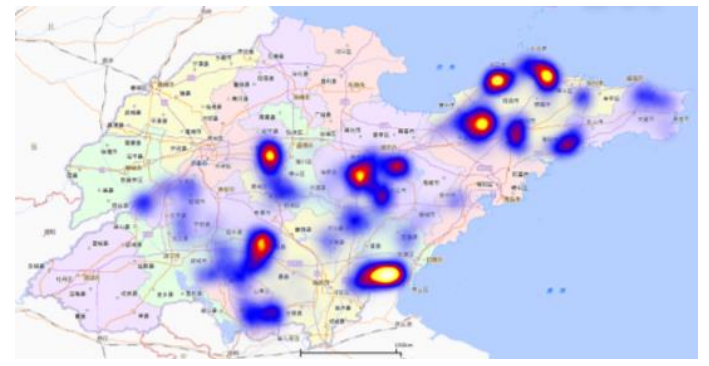

Figure 7. Map of illegal mining prone spots between 20132016

\section{CONCLUSIONS}

Remote sensing dynamic monitoring information system of mineral development is successfully built by integrated application of geographic information system, global positioning system and mobile Internet technology tightly around the actual application needs of mine remote sensing dynamic monitoring, and is practically applied in Shandong Province. The establishment of the system effectively improves the timeliness and accuracy of remote sensing dynamic monitoring in Shandong Province. Rapid response mechanism of "fast data acquisition, timely target detection, fast handling and timely feedback" is constructed, and transformation from traditional manual supervision to informational monitoring is achieved, thereby improving the efficiency of mining legal supervision and enhancing the informational management level of land and resource authorities, which are of great significance for the establishment of good mineral development order, promotion of safety production, and protection of mining ecological environment in Shandong Province.

\section{REFERENCES}

Datcu, M. , 2003. Information mining in remote sensing image archives: system concepts. IEEE Trans.Geoscience and Remote Sensing, 41(12), pp.2923-2936.

Nie Hongfeng, 2007. The problems in the remote sensing monitoring technology for the exploration of mineral resources and the counter measures. Remote Sensing for Land \& Resources, 74(4),pp.11-13.

Chen Weitao,2009. Advances in remote sensing-based detecting of mine explotation and mine environment.Remote Sensing for Land \& Resources,80(2),pp.1-8.

Nan Junxiang, 2012. Remote sensing investigation of coal mines in Xuanwei of Yunnan province for their
development.Remote Sensing for Land \& Resources,93(2),pp.121-124.

Wang Yonggang, 2008. Remote sensing and GIS technology based monitoring of the mineral exploitation in Beijing area.Land and Resources Informatization,6,pp.6-11.

Cao Hongsong,2007. Study on application of remote sensing and GIS technologies in dynamic monitoring in cangshan mine exploration.Shandong Land and Resources,23(67),pp.43-47.

National Geomatics Center of China, 2011. Basic Geomatics Information Center of Heilongjiang, Integrated geographic information center of Shanxi Province,etal..CH/Z90112011,Platform for geo- -information common services Data specification for electronic map. Beijing: Surveying and mapping press.

$\mathrm{Yu}$ Tao, 2002. Mobile computing environment the development and application of GIS technology.Bulletin of Surveying and Mapping,2,pp.40-42.

Zhang Ergang, 2013. Research and implementation of data acquisition system based on mobile GIS technology. AnHui University of Science and Technology,pp.6-8.

Xu Liuhua, 2012. The research and experiment on the mobile field data collection system based on iPad. Bulletin of Surveying and Mapping,12,pp.75-78.

Li Yao, 2012. Design and Implementation of Picture Information Acquisition Management system based on Exif.Computer and Modernization, 9,pp.134-1. 